\title{
SINGLE-LAYER ELASTOMERIC OUT-OF-PLANE ACTUATOR WITH ASYMMETRIC SURFACE PROFILE
}

\author{
T. Maleki ${ }^{1,3}$, G. Chitnis ${ }^{2,3}$, A. Panja ${ }^{1,3}$, B. Ziaie $^{1,3}$ \\ ${ }^{1}$ School of Electrical and Computer Engineering \\ ${ }^{2}$ School of Mechanical Engineering \\ ${ }^{3}$ Birck Nanotechnology Center, \\ Purdue University, West Lafayette, IN, USA
}

\begin{abstract}
In this paper, we report on the first batch-fabricated singlelayer elastomeric out-of-plane actuator using an asymmetric surface profile. The cantilever actuator is composed of a single polydimethylsiloxane (PDMS) layer and soft-lithographically patterned conductive carbon grease electrodes. Low actuation voltage and large out-of-plane displacement are achieved using a flat surface on one side and a corrugated one on the opposite side. An actuator $5 \mathrm{~mm}$ in length, $1 \mathrm{~mm}$ in width, and $80 \mu \mathrm{m}$ in thickness can produce up to $650 \mu \mathrm{m}$ out of plane displacement. The same actuator consumes $20 \mu \mathrm{W}$ of static power and generates $4 \mu \mathrm{N}$ of force.
\end{abstract}

\section{INTRODUCTION}

Out-of-plane actuators are essential elements in micropumps, micromirrors, and many other MEMS devices. Many actuation mechanisms (electrostatic, magnetic, piezoelectric, etc.) have been used to implement such structures, each with its unique advantages and disadvantages. Elastomeric actuators (or dielectric elastomer DE actuators) are an emerging class of electromechanical transducers having the ability to emulate biological muscle in achieving high fracture toughness, large strain, and inherent vibration damping [1]. Major efforts in this area have been devoted towards reducing the actuating voltage and increasing the strain through various mechanical modifications. Although some out-of-plane actuators using dielectric elastomers have been reported, they are mostly hand-made, require several elastomeric layers, and have complicated fabrication processes involving stretch-frames and pre-patterned dome-shape elastomers [2, 3]. These complicated fabrication processes are not batch-compatible which increases their cost and makes their integration into a MEMS system very cumbersome. In this paper, we report on the first batch-fabricated single-layer elastomeric out-of-plane actuator using an asymmetric surface profile. The actuator uses a geometric design to achieve a single layer configuration. In addition, soft lithography and laser-micromachining are used to batch fabricate the actuators.

\section{DESIGN AND SIMULATION}

Figure 1 shows 3D schematic and cross-section of a singlelayer elastomeric cantilever actuator with an asymmetric surface profile, i.e., the top surface is corrugated while the bottom one is flat. As can be seen in the Figure 1b, the thickness (d) of the actuator is not constant. It can be modeled as two components, a constant $(\mathrm{t})$ and a square wave (magnitude $A$ and frequency $\omega$ ) representing the corrugation. Upon actuation, the elastomer undergoes a transverse contraction given by

$$
\frac{\Delta d}{d}=-\frac{1}{2 E} \varepsilon \varepsilon_{0} \frac{V^{2}}{(t \pm A)^{2}}
$$

where $d$ is the thickness of the elastomer, $E$ is its Young's modulus, $\varepsilon$ is its dielectric constant, $\varepsilon_{o}$ is the air permeability, and $V$ is the applied voltage. Due to the asymmetric surface profile, a non-uniform transverse compression and longitudinal expansion is created, inducing a bending moment in the cantilever.
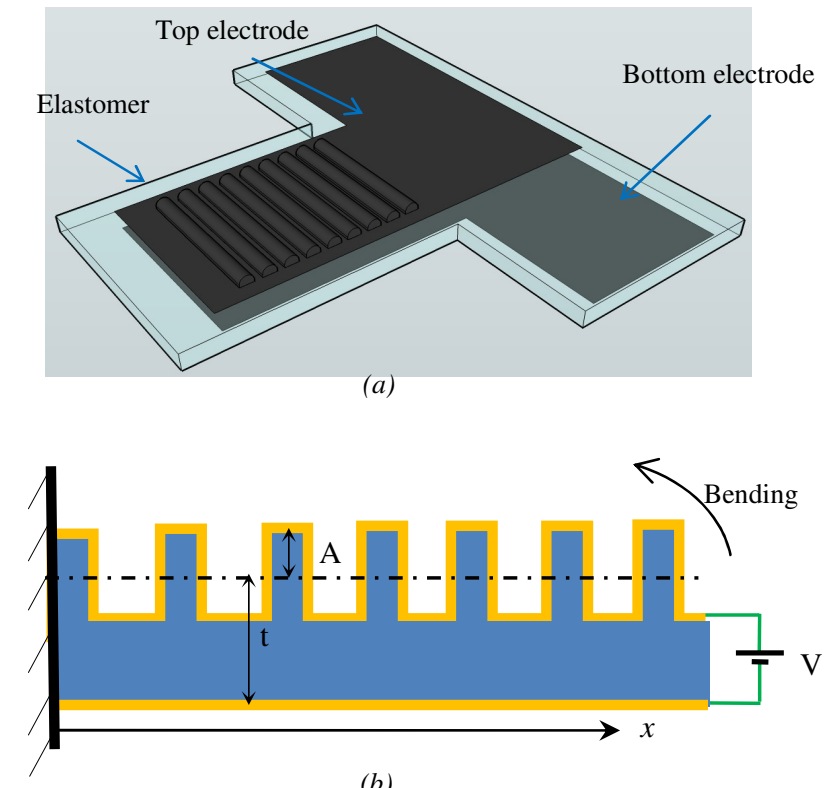

(b)

Figure 1: 3D schematic (a) and cross section (b) of a singlelayer elastomeric actuator with an asymmetric surface profile.

Figure 2 illustrates a simplified structure in order to better understand the actuation mechanism. This simplified model assumes the induced electrostatic force is perpendicular to the surface of the actuator. Figure $2 a$ represents a single actuator element without any actuation voltage, while similar element after actuation is depicted in Figure $2 b$. As can be inferred from Equation 1, the induced attraction force is larger in the thinner segment as compared to the thicker segment, resulting in an asymmetric compressive strain in the element. The thicker area of the element can be divided into two distinct parts, Figure $2 b$. The lower part (darker color) that is connected to the thinner segment experiences higher transverse strain compared to the top part. Since PDMS elastomer has a Poison's ratio close to 0.5 , this transverse strain induces a longitudinal strain, hence expanding the bottom part more than the top. This asymmetric longitudinal expansion creates a unimorph actuator, bending the cantilever beam in the upward direction. 
(a)

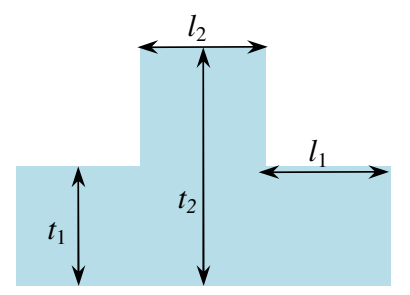

(b)

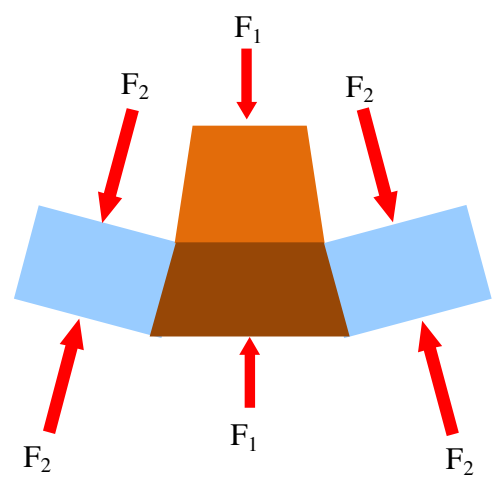

Figure 2: Simplified structure of one element of the asymmetric actuator. (a) without applied voltage, (b) with applied actuation voltage inducing a bending moment in the element

$\mathrm{Comsol}^{\circledR}$ Multiaphysics was used to simulate the stress/strain in an $5 \mathrm{~mm}$ long, $1 \mathrm{~mm}$ wide, PDMS $(\mathrm{E}=750 \mathrm{kPa}, \varepsilon=2.7)$ actuator having different corrugation structures $\left(l_{l}, l_{2}, t_{l}, t_{2}\right)$. Figure 3 shows the simulation result for an actuator with corrugation parameters of $l_{l}=60 \mu \mathrm{m}, l_{2}=20 \mu \mathrm{m}, t_{l}=40 \mu \mathrm{m}$, and $t_{2}=100 \mu \mathrm{m}$ under $1 \mathrm{kV}$ applied voltage (left side is the fixed point). The inset in the figure clearly proves our hypothesis that the induced transverse stress is not uniform through the thicker part of the PDMS element causing a bending moment in the actuator.

Figure 4 represents the deflection simulation results versus thick segment's length $\left(l_{2}\right.$ in figure $\left.2 a\right)$ for a $5 \mathrm{~mm}$ long, $1 \mathrm{~mm}$ wide actuator when $1 \mathrm{kV}$ voltage is applied. In this simulation, the thickness of the thin segment $\left(t_{l}\right.$ in figure $2 a$ ) and the whole element length $\left(l_{1}+l_{2}\right)$ are assumed to be fixed at $40 \mu \mathrm{m}$ and $80 \mu \mathrm{m}$, respectively. As can be seen, deflection is increasing with larger $l_{2} / l_{1}$ ratio. This can be explained by the fact that the induced deflection is mostly due to an asymmetric strain in the thicker segment (see the inset of Figure 3), and the length of the thin segment has limited effect on the deflection. Therefore, the longer the thick segment, the higher the deflection. The inset in Figure 4 shows deflection versus thick segment thickness, $t_{2}$, while $l_{l}, l_{2}$, and $t_{l}$, are held constant at $5 \mu \mathrm{m}, 75 \mu \mathrm{m}$, and $40 \mu \mathrm{m}$, respectively. As illustrated in the figure, deflection is higher for an actuator with increased thick segment thickness, due to the minimal effect of thin segment width on the asymmetry of the induced longitudinal strain.

Simulation results for deflection versus thick layer length for $5 \mathrm{~mm}$ long, $1 \mathrm{~mm}$ wide actuators having different thin segment thickness, $t_{1}$, is depicted in Figure 5. In this simulation, the thickness of the thick segment, $t_{2}$, is assumed to be fixed at $100 \mu \mathrm{m}$. As shown in the figure, the thinner the thin segment, the higher the deflection. This is because of the higher transverse contraction (see Equation 1) in the thin segment. The inset of the figure represents the deflection versus thin segment thickness, $t_{l}$, while $l_{l}$, $l_{2}$, and $t_{2}$, are $5 \mu \mathrm{m}, 75 \mu \mathrm{m}$, and $100 \mu \mathrm{m}$, respectively.

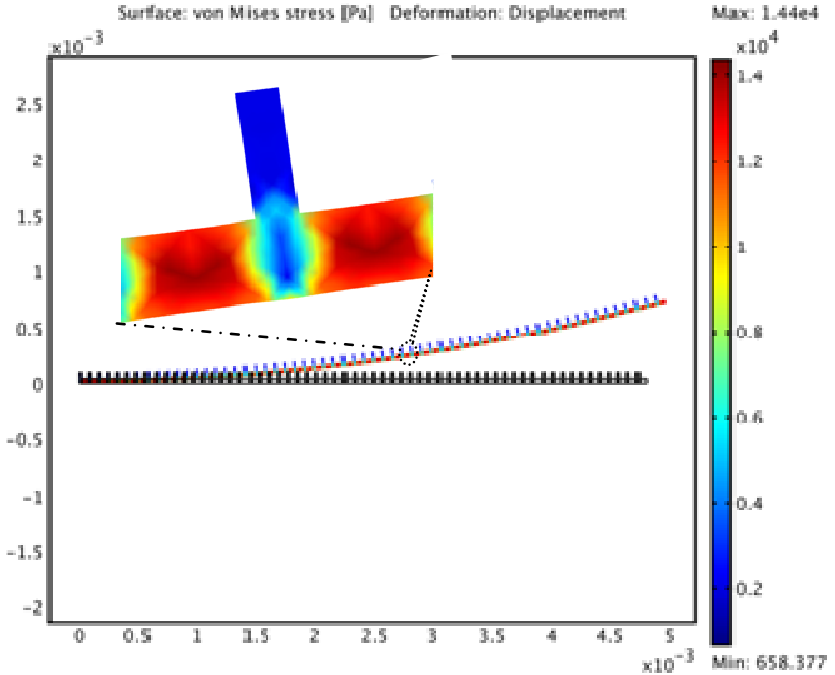

Figure 3: Comsol ${ }^{\circledR}$ simulation results for the new asymmetric actuator. Inset clearly shows non-uniform induced stress in the thicker segment.

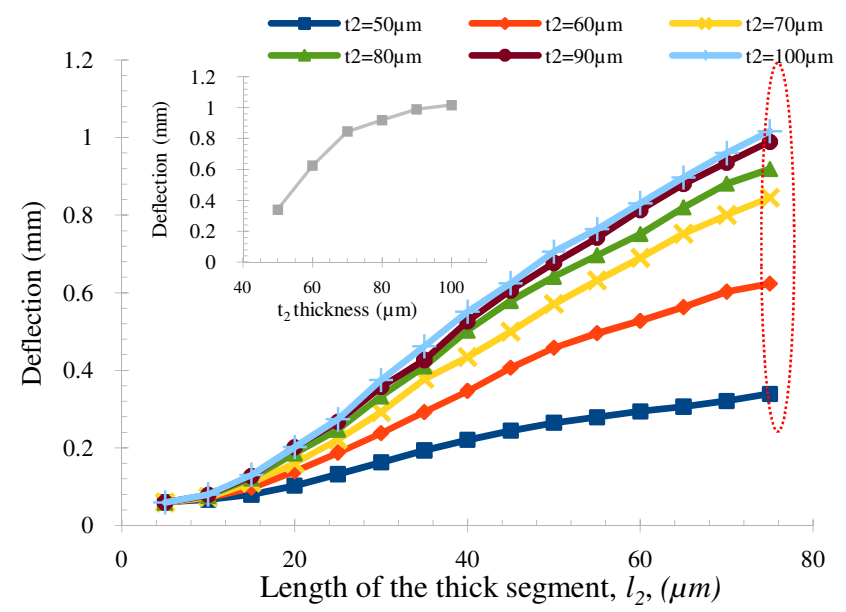

Figure 4: Simulation results for asymmetric actuator having a constant thin segment thickness and various other geometries.

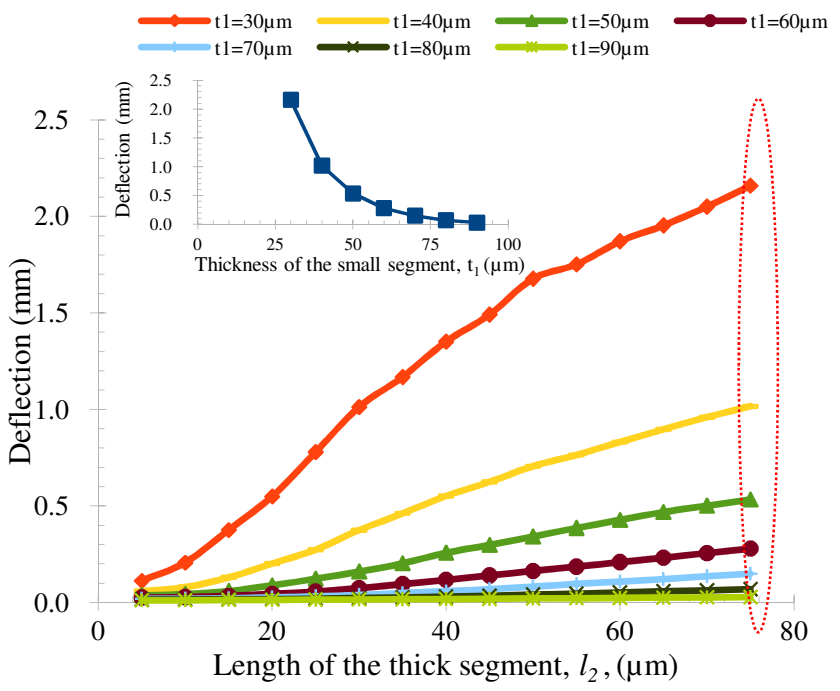

Figure 5: Simulation results for an actuator with constant thick segment thickness and various other geometries. 
In another set of simulations, the end point of the actuator with $40 \mu \mathrm{m}$ thin segment and $100 \mu \mathrm{m}$ thick segment was fixed and the reaction force at that point was calculated. The results of this simulation are plotted in Figure 6. As shown in the figure, an actuator with a longer thick segment creates less force. This is due to the fact that longer segment can dissipate nonsymmetrical strain in itself by less amount of force that a short segment.

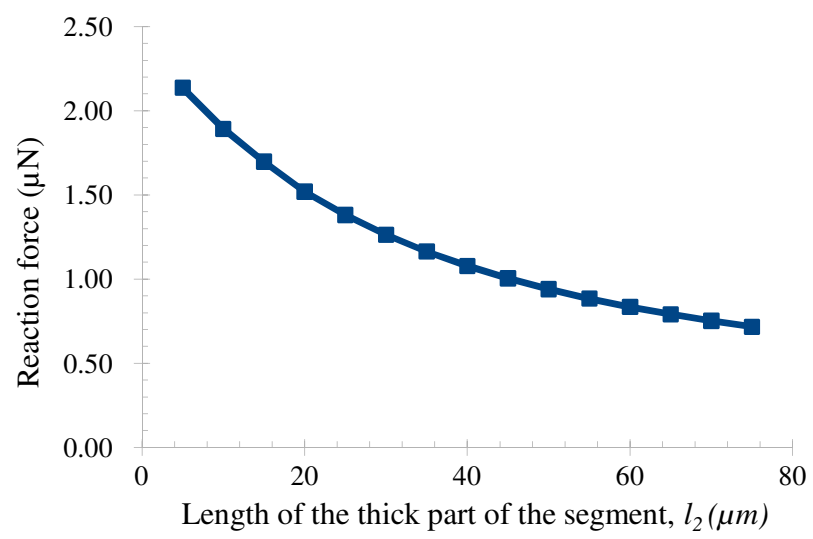

Figure 6: Reaction force at the end of actuator when the end point if fixed.

In conclusion, simulation results suggest that an actuator with a very asymmetric profile creates the maximum deflection. However, such an actuator would generate least amount of force. We should mention that practical limitations might prevent the fabrication of such structures. For example, in an actuator with very narrow and deep surface non-uniformity, achieving a uniform electrode coating is very difficult. Moreover, handling ultra thin PDMS sheets (less than $40 \mu \mathrm{m}$ ) is another challenge in fabricating such an actuator. In the following sections, fabrication and experimental results for an actuator with following geometrical parameters is presented, $l_{l}=20 \mu \mathrm{m}, l_{2}=70 \mu \mathrm{m}, t_{l}=40 \mu \mathrm{m}$, and $t_{2}=100 \mu \mathrm{m}$.

\section{FABRICATION PROCESS}

Figure 7 shows the fabrication process for an elastomeric actuator using PDMS as the structural material and conductive grease as the electrodes. An advantage of using PDMS is its well known mechanical properties, low cost, and MEMS compatible fabrication process-ability [4]. Conductive carbon grease was chosen as the electrode material due to its good adhesion to PDMS and low deformation resistance.

The process starts with the fabrication of a silicon mold with a corrugated top surface $(60 \mu \mathrm{m}$ deep) using HNA (20\%wt HF:70\%wt $\mathrm{HNO} 3: 10 \%$ wtCH3COOH) as the etchant and silicon nitride as mask, Figure $7 a$. Next, a $70 \mu \mathrm{m}$ thick PDMS (SYLGARD 184, Dow Corning, Midland, MI) is spun coated (1500rpm for $30 \mathrm{sec}$ ) and cured (at $120{ }^{\circ} \mathrm{C}$ for $30 \mathrm{~min}$ ) to form the structural layer, Figure $7 b$. Subsequently, an electrode stamp is fabricated by casting PDMS against a SU8-on-silicon mold, Figure 7c. The stamp is inked by conductive carbon grease (MG Chemicals, Burlington, Ontario, CA), and is used to deposit the top electrode on the PDMS layer, Figure $7 d$. The stamped PDMS layer is then separated from the mold and transferred to a parylenecoated silicon wafer. This is followed by the aligned stamping of the top electrode on the corrugated surface, Figure $7 e$. A slight pressure is applied during this step in order to deform the corrugations and ensure a uniform coverage. Finally, individual actuators are separated at the wafer level using laser micromachining, $\left(\mathrm{CO}_{2}\right.$ laser generated by 2007 Professional Systems from Universal Laser Systems Inc. AZ, USA), Figure $7 f$.
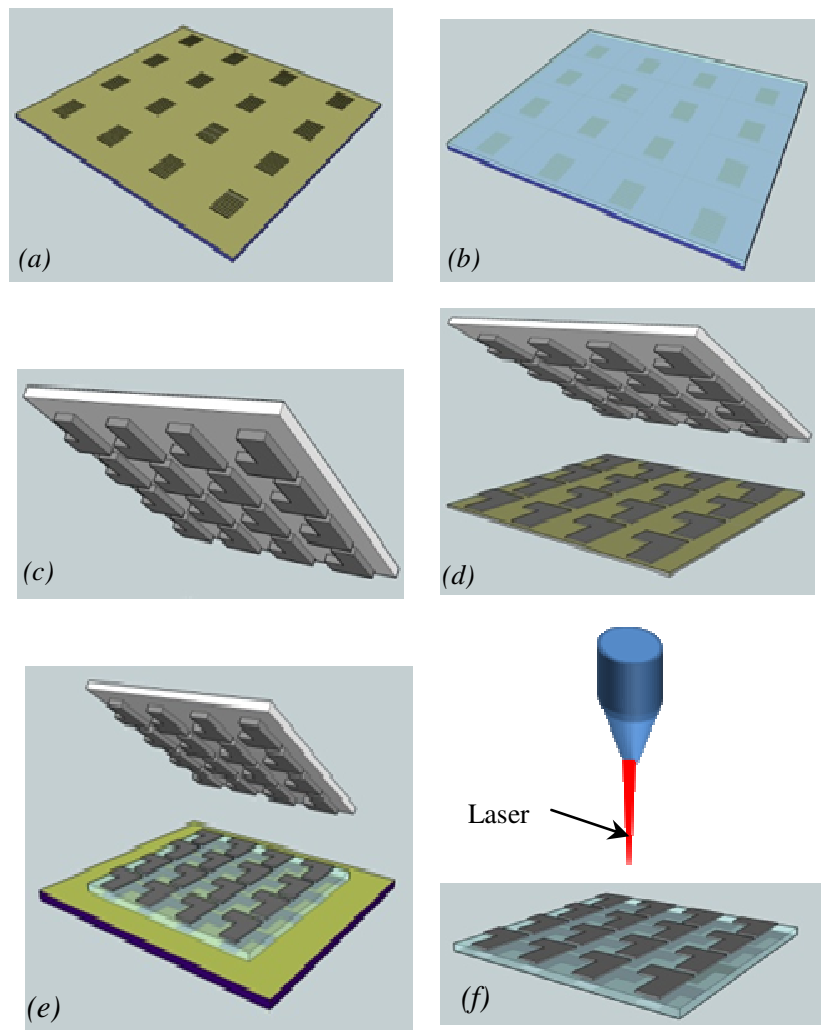

Figure 7: Batch-scale fabrication process of elastomeric actuators.

Figure $8 a$ shows a wafer with batch fabricated actuators prior to separation. SEM picture of the actuator illustrating the uniform coverage of the corrugated surface by carbon grease is depicted in Figure $8 b$. Figure $8 c$ is a close-up optical photograph of the mounted actuator showing the corrugations. Figure $8 d$ demonstrates the testing setup in which the actuator is mounted on a metal-coated glass slide acting as the backside connection and a needle used as the front contact.

\section{MEASUREMENT RESULTS}

The actuators were tested using a high voltage power supply and their displacement was measured through a video framegrabber. Figure 9 shows the tip-displacement vs. applied voltage for three different lengths $(3,4$, and $5 \mathrm{~mm})$. One should notice that the measured displacement for a $5 \mathrm{~mm}$ actuator is well matched the simulation result (see figure $5, t_{1}=40 \mu \mathrm{m}$, and $l_{2}=70 \mu \mathrm{m}$ ). As can be seen, mm scale actuation was achieved for voltages of less than $1 \mathrm{kV}$. The maximum power for a $5 \mathrm{~mm}$ long actuator with $900 \mathrm{~V}$ actuation voltage was $20 \mu \mathrm{W}$.

In another experiment, the required force to bend the unactuated cantilevers is measure and results are plotted in Figure 10. As depicted in the figure, $\mu \mathrm{N}$ force is required to generate $\mathrm{mm}$ bending in the cantilever. By comparing figures 9 and 10, one can calculate the zero-deflection force of the actuators. 

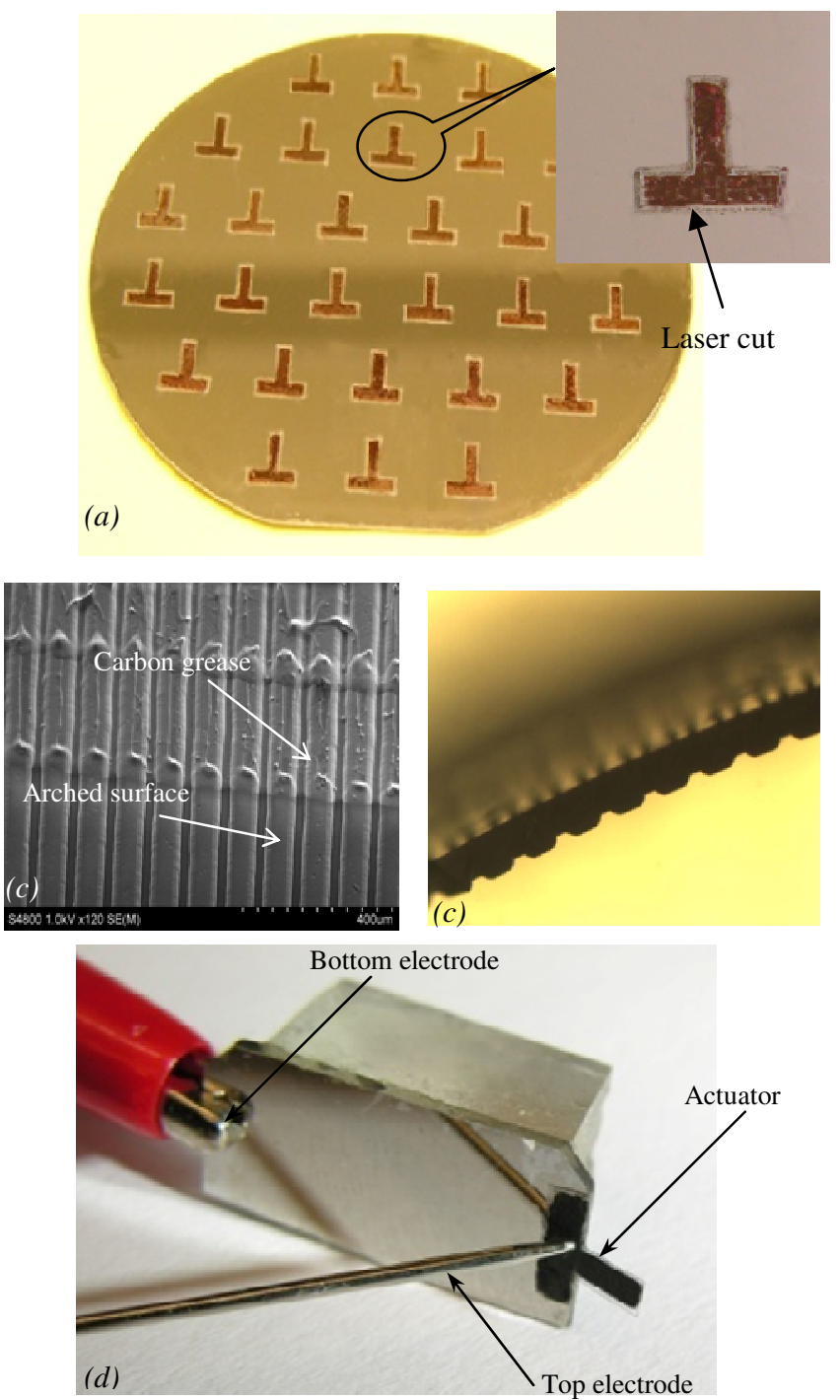

Figure 8: Batch fabricated actuators, b) SEM picture of the single layer actuator, c) cross sectional photograph of the actuator, d) a mounted actuator.

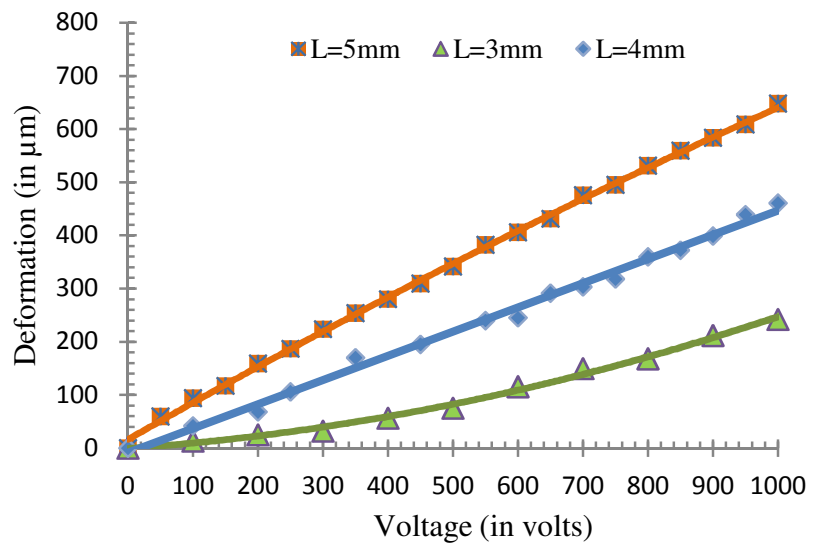

Figure 9: Microactuator displacement vs. voltage $\left(l_{1}=20 \mu \mathrm{m}\right.$, $l_{2}=70 \mu \mathrm{m}, t_{1}=40 \mu \mathrm{m}$, and $t_{2}=100 \mu \mathrm{m}$.).

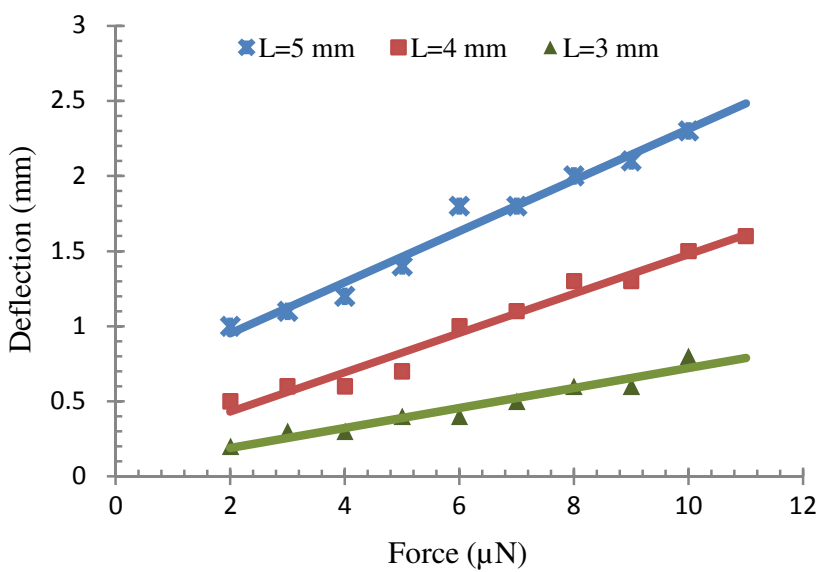

Figure 10: Deflection vs. applied force for $1 \mathrm{~mm}$ wide actuators with different length $\left(l_{l}=20 \mu \mathrm{m}, l_{2}=70 \mu \mathrm{m}, t_{l}=40 \mu \mathrm{m}, \quad\right.$ and $t_{2}=100 \mu \mathrm{m}$.).

\section{CONCLUSIONS}

In this paper, we presented a batch fabrication process for the first single layer elastomeric actuator based on asymmetric surface profile that can achieve large out-of-plane deflections with a low actuation voltage. Comsol $^{\circledR}$ simulation results showed that actuators with highest non-uniform profile creates the maximum deflection. However practical limitations prevented fabrication of such structures. Three different size actuators with optimized geometry for maximum out-of-plane deflection were successfully fabricated and characterized. Their measured performances showed a good agreement with the $\mathrm{Comsol}^{\circledR}$ simulation results. Millimeter scale out of plane displacement was achieved using a fairly low voltage (less than $1 \mathrm{kV}$ ). A $5 \mathrm{~mm}$ long actuator was capable of generating $5 \mu \mathrm{N}$ force when actuated with $1 \mathrm{kV}$ voltage and consumed less than $20 \mu \mathrm{w}$ power.

\section{REFERENCES}

[1] A. O'Halloran, F. O'Malley, P. McHugh, "A review on dielectric elastomer actuators, technology, applications, and challenges", Journal of Appl. Phys. (104), 071101, pp. 1-10, 2008.

[2] R. Kornbluh, R. Pelrine, Q. Pei, R. Heydt, S. Stanford, S. Oh, J. Eckerle, "Electroelastomers: applications of dielectric elastomer transducers for actuation, generation and smart structures" Proceedings of the SPIE, (2002), pp. 254-270.

[3] F. Carpi, G. Frediani, A. Mannini, D. De Rossi "Contractile and buckling actuators based on dielectric elastomers: devices and applications", Advances in Science and Technology, 61, pp. 186-191, 2008.

[4] X. Q. Brown, K. Ookawa, J. Y. Wong, "Evaluation of polydimethylsiloxane scaffolds with physiologically-relevant elastic moduli: interplay of substrate mechanics and surface chemistry effects on vascular smooth muscle cell response" Biomaterials, 26 (16), pp 3123-3129, 2005.

\section{CONTACT}

*B. Ziaie, tel: +1-765-494-0725; bziaie@purdue.edu 